\title{
Explaining the Choice of Distribution Rule: The Role of Mental Representations*
}

\author{
Rosamund E. Stock, London School of Economics
}

\begin{abstract}
Justice researchers have long puzzled over the enormous variation in the distribution rule considered appropriate in different situations, and no overall pattern of rule use has yet been identified. A series of in-depth, semistructured interviews was combined with a small-scale $(N=229)$ survey carried out in the UK to test the hypothesis that it is the perception of the situation that mediates the use of justice rules. Content analysis revealed significant associations between the distribution rules mentioned or endorsed spontaneously and the descriptions of groups, social relations, and people that the subjects used in prompted and unprompted explanations. These different elements associated with a rule form a "justice ideology" and constitute a representation of society. The rules used were different for different resources within a domain, calling into question the existence of a single, domain-specific rule. This sample of British respondents was firmly in favor of a more egalitarian distribution of income and favored the use of a need rule in the local community.
\end{abstract}

\section{Introduction}

This paper seeks to do three things: to address a gap in the literature, to synthesize a body of theoretical material, and to test hypotheses derived from this theoretical framework.

There has not been a huge amount of qualitative work on distributive justice. Hochschild's (1981) study of American respondents is still the largest and best known. Kellerhals, Coenen-Huther, and Modak (1987, 1988) and Matthews (1987) look at families, but no detailed work exists for British respondents. It is commonplace in this field of research that different rules are used for different social situations (such as work, intimate relationships, and the family), by different groups (such as young people versus business people), and by different cultures. As a result, one cannot assume that results from America and other European respondents can be translated.

There has been considerable convergence in thinking on the psychology of distributions. There are roughly three broad distribution rules (or principles of justice), associated with different resources, domains of life, and social relationships. This multiprinciple approach (Tornblom 1992) to distributive justice has always had a problem with the absence of a clear pattern in the choices people make or advocate in different situations and for different resources. A 
comparison of studies from different cultures comparing groups within them (Tornblom and Foa 1983) could identify no regularity in which rules were used for which situations, and it concluded that the prediction that different rules would be used for different types of social relation had not been realized. Is it worth re-evaluating this approach?

\section{Theoretical Background}

Justice research has usually investigated the use of three broad "principles of justice": proportionality (a differential distribution, usually according to some criterion), equality, and need. There have, however, been several formulations of the multiprinciple approach. This study makes use of three. Deutsch's (1985, 1987) experimental work gave rise to his "crude law of social relations." This states that the typical consequences of a social relationship (here seen as a mode of cooperation or interaction between social actors), such as the choice of a distribution rule consonant with that type of relationship, can give rise to that form of social relations: people working in a cooperative regime tend to choose equality as the appropriate distribution rule, and those working under an egalitarian reward structure tend to become cooperative. The different distributions are part and parcel of fundamentally different orientations, manifest in both attitudes and behavior to others in a group.

Lerner's (1991) developmental approach describes the use of different distribution rules as a child grows up. The use of different rules is associated with the development of specific forms of social relationship. At first, the child does not differentiate between self and other (identity relations), and need is the overriding rule. Then the child learns to discriminate between self and other (but no further), and unit relations are associated with equality (treating the same as the same). Lastly, nonunit relationships between units, differentiated as bundles of varying characteristics, are associated with proportionality.

The idea that social relationships are somehow integrally bound up with distributions of goods received some useful theoretical development from Fiske's (1991) theories of the structure of social relationship. This postulated the existence of four basic types of social relationship, each with its typical form of social exchange (both distribution and contribution), symbolic marking, definition of persons, mode of decision-making, and form of punishment. The four basic relationships can be combined, iterated, and nested to give rise to more complex relationships, but the basic building blocks are seen as universal. The knowledge needed to operate such different types of relationship is thought to be lodged in a cognitive unit referred to as a "schema," and the operation of this schema gives rise to the qualitatively different types of relationship.

Fiske's (1991) theory also postulates that the operation of these cognitive structures gives rise to the experience of obligation. Essentially, the schema 
mechanism provides a formal transformation rule that orders incoming perceptions of the social field by telling the observer what ought to go with what. The schemata are similar to the "justice ideologies" suggested by Kayser and Schwinger (1982) for ordering the social information necessary to make allocation decisions. They differ from the traditional view in justice research, however, which sees the distribution rule as separate from the standard of evaluation (used to make fairness judgments of a particular allocation; see, e.g., Cohen 1987). Lerner, too, regards the primitive scripts underlying the different types of relationship that appear in childhood as distinct from socially learned rules (Desmarais and Lerner 1994). In contrast, Fiske's schemata are both prescription for use and standard of judgment, expectation, and obligation: through their influence on the behavior of social actors, they are incorporated into culture, while the surrounding culture also shapes the cognition of individuals.

All of these approaches stress that different distribution rules will be associated with the measurable manifestations of fundamentally different types of social relationships, and that these relationships will be experienced as qualitatively different. However, it must be remembered that this is a profoundly psychological phenomenon: Abric and Kahan (1972) criticized Deutsch's experimental approach for failing to distinguish between the actual characteristics of the experimental situation and the way participants saw it. Perhaps the answer to the apparent lack of association between choice of allocation rule and the type of social relationship (Tornblom and Foa 1983) is to seek it in perceptions of the situation. It is in these perceptions that the relationship schemata will function.

From this, we hypothesize that there will be significant associations between characteristics of the social relationship (the mode of interaction) and the distribution rule. We further hypothesize that there will be a set of attitudes associated with different distribution rules, and that these elements will constitute some kind of description of a social relationship.

\section{Methods}

Twenty-one in-depth interviews were undertaken with twenty-two subjects (a second respondent joined in one interview, which was retained because it was very productive of relevant material). Respondents came from the southeast and northwest of England. The latter was a small subsample used simply to investigate the possibility of subcultural variation. Interviews were transcribed and content-analyzed with a coding frame that started with the attitudes outlined above: descriptions of people, groups, and social relationships as cooperative, competitive, and nurturant. The rest of the coding frame was developed from the interview material and coded for distribution mechanism, individualistic, and hierarchical elements. 
The text was split into units by subject matter. Everything that the respondent said about a particular topic at that point in the interview was kept together and considered to be a single construction of the social field. When either a new question was asked or the respondent began speaking of something else, a unit boundary was created. Each unit was classified by domain (a broad, institutionally defined, and socially recognized area of life) and resource (specific social objects to be distributed, including such abstractions as "treatment by others" and "job opportunities").

The units were pooled into a single database of $N=1,785$. The rationale for doing this is that people do not develop their ideas de novo, but are immersed in a culture, which gives them many of the building blocks of their ideas, any of which may have been used by any of the respondents. Crosstabulation of rule use by respondent showed that there was more variation in the rules mentioned within the replies of one respondent than between respondents.

From the interview texts, a questionnaire was prepared that asked respondents about the distribution of income and one other social resource, and distribution in one other domain of life (the local community or the family). This self-completion questionnaire was distributed at specific workplaces and delivered to houses in a borough in northeast London. The sample was not representative, and external circumstances determined its availability. The data were entered into a data matrix for the SPSS (v. 7) package.

\section{Qualitative Results}

\section{Use and Endorsement of Distribution Rules among British Respondents}

Each instance of a distribution rule was coded for the type of rule used, proportionality, near equality (mentioning reduced differentials), equality, and need. The evaluation given (positive, neutral, ambivalent, or negative) was also recorded.

Proportionality was the default rule, and was also recorded when a respondent mentioned only a basis (criterion) for a rule. The results for the criteria will not be considered here. (See Table 1.)

The proportionality rule predominates in the social domain and at work. In both domains, actual endorsement (that is, mention with a positive evaluation) is less than the number of times mentioned. But the overriding impression is of a differential distribution, which is largely accepted in the work domain but about which there is dissensus in the social domain.

The local community shows much decreased use of proportionality and the highest use of need; the use of proportionality is the lowest for any domain. A result of this type has not been reported in any other data; thus, it represents a finding unique to British subjects. However, it should be borne in mind that the 
Table 1

Rule Use by Domain

Percentage of rules mentioned in each domain (independent of evaluation); that is, percentage of the number of mentions of distribution rules accounted for by each rule.

$\begin{array}{lcccr} & \text { PROP } & \text { NREQ } & \text { EQ } & \text { NEED } \\ \text { Society } & 68.5 & 3.7 & 14.2 & 13.5 \\ \text { Work } & 75.9 & 3.2 & 13.4 & 7.5 \\ \text { Local community } & 31.5 & & 21.9 & 46.6 \\ \text { Voluntary social life } & 52.5 & 1.7 & 40.7 & 5.1 \\ \text { Family } & 42.0 & & 34.8 & 23.2\end{array}$

Percentage of endorsed rules by domain; that is, percentage of those rule mentions where the rule was positively evaluated accounted for by each rule.

$\begin{array}{lcccc} & \text { PROP } & \text { NREQ } & \text { EQ } & \text { NEED } \\ \text { Society } & 50.1 & 6.0 & 22.5 & 21.4 \\ \text { Work } & 64.3 & 1.2 & 23.8 & 10.7 \\ \text { Local community } & 9.8 & & 29.4 & 60.8 \\ \text { Voluntary social life } & 28.2 & 2.6 & 61.5 & 7.7 \\ \text { Family } & 26.5 & & 44.1 & 29.4\end{array}$

lead question in this domain was about local authority resources in times of scarcity, and was often prompted with questions about distribution between, for example, social services and education. This result may overstate the case slightly, as need is known to be a favored rule in times of scarcity (Skitka and Tetlock 1992).

Equality is favored in the voluntary domain; use of proportionality is divided between positive and negative evaluations. However, personal circumstances are mentioned as a basis of proportionality, whereas status is explicitly disapproved. In other words, people favor a distribution based on personal characteristics and reject a hierarchical one. A similar pattern appears for the family domain. However, the consideration of which resources within a domain attract which rules changes the picture entirely.

The detail of the way in which rule use and endorsement vary across resources does not support the idea of a dominant rule for a domain. Can one really say, looking at Table 2, that there is one dominant rule, even for society? Furthermore, even where a particular rule is used or endorsed most of the time, 
Table 2

Percentage of Rule Use and Endorsement for the Most Frequently Mentioned Resources

\begin{tabular}{lcccc}
\hline & PROP $^{\mathrm{a}}$ & NREQ $^{\mathrm{a}}$ & EQ $^{\mathrm{a}}$ & NEED $^{\mathrm{a}}$ \\
& & & & \\
\hline Income & $70.5(58.3)$ & $6.3(9.5)$ & $9.4(12.7)$ & $13.9(19.4)$ \\
Health care & $51.1(25.9)$ & $2.1(3.4)$ & $11.3(17.2)$ & $35.1(53.4)$ \\
Educational opportunities & $55.3(10.0)$ & $4.3(5.0)$ & $23.4(50.0)$ & $17.0(35.0)$ \\
Job opportunities & $71.6(46.3)$ & $1.0(2.4)$ & $23.5(46.3)$ & $3.9(4.9)$ \\
Treatment & $76.6(49.2)$ & & $21.6(47.7)$ & $1.8(3.1)$ \\
Pay (work) & $78.7(88.0)$ & $9.8(4.0)$ & $6.6(0)$ & $4.9(3.6)$ \\
Treatment at work & $68.8(53.6)$ & & $27.1(42.9)$ & $4.2(3.6)$ \\
Promotion & $88.9(87.5)$ & & $3.7(12.5)$ & $7.4(0)$ \\
Other res. at work & $72.0(40.9)$ & & $14.0(31.8)$ & $14.0(27.3)$ \\
& & & & \\
\hline a $\%$ use (+ve). & & & & \\
\hline
\end{tabular}

the level of use of other rules is still substantial. For example, proportionality is used 70 percent of the time for income, but represents only about 60 percent of positive rule use. Thirty-three percent of rule endorsements are for need or equality. This is a large degree of dissensus. There is also the issue that people may talk entirely differently when they are considering the tax and benefit system than when they are considering "earned income," just as the picture changes when income is framed in the social and work domains.

\section{Association of Rule Use with Descriptions}

The question then becomes, is there any significant association between the rules used and the way the situation is being perceived and described? Table 3 examines the use of different descriptions of the group or society. Clearly, some categories are used more often with some rules than others. When proportionality is used, a respondent is more likely to describe the group or society as hierarchic; when need is used, a respondent is more likely to describe it as nurturant. It should be remembered that these descriptions comprise any mention within the same social field, not necessarily one explicitly connected by the subject.

It is evident that very different elements are associated with different rules. The association of descriptions of distribution mechanisms, groups, social relations, and individuals (unevaluated) with rule is significant $(\mathrm{p}<.0001)$, and the 
Table 3

Frequency of Group Categories by Rule

\begin{tabular}{lrrrr}
\hline & PROP & NREQ & EQ & NEED \\
\hline Hierarchic & 81 & 6 & 8 & 3 \\
Community & 14 & 5 & 13 & 3 \\
Cooperative & 2 & & 2 & \\
Competitive & 8 & & 1 & 15 \\
Nurturant & 6 & & 2 & 2 \\
Aggregate & 1 & & & 2 \\
Organic & 1 & 1 & & \\
Oppressive & 7 & & & \\
Structured & 1 & & & \\
& & & & \\
\hline
\end{tabular}

association is mainly accounted for by positive and neutral mentions ( $\mathrm{p}<$ .0001). The frequencies for rule endorsement are actually somewhat less clear.

If, for each rule, we identify the most frequently used category of descriptive element (a difference of $\leq 5$ between the frequency of the chosen category and the next most frequently used), we obtain the information shown in Table 4. These sets of elements make good candidates both for justice ideologies and for relationship schemata. Each describes a different sort of group. When a similar analysis is made of these data using the four-rule structure advocated by Fiske (1991) (coding the fourth authority-ranking rule as proportionality based upon status), no additional discrimination occurs between descriptive categories.

\section{Survey Results}

The small-scale survey $(N=229)$ replicated some of the results above. The very different demand characteristics of a self-completion survey do not always permit direct comparison. Both descriptive ("is") and normative ("should") statements were supplied for the distribution of income, using the proportionality, need, and equality rules, and the near-equality rule flagged in the qualitative data.

Table 5 shows the percentage of respondents agreeing or strongly agreeing that the four different distribution rules are currently applied principles for the distribution of income. Clearly, people see a proportional distribution (large differentials) and - somewhat in contrast to other data (e.g., Kelley and Evans 1991) - most respondents do not recognize need to be a currently used distribution rule. The endorsement of near equality ("the income people get should vary but 
Table 4

Elements Associated with Rule Use

\section{PROPortionality:}

- Hierarchical group

- Individuals as differentiated

- Market distribution mechanism

- Poor/uncooperative social relations

\section{EQuality:}

- Group as community

- Individuals defined as similar or different

- Cooperative social relations

- System or organization as distribution mechanism

\section{NEED:}

- Nurturant group

- Individuals as similar, with basic needs and wishes or as needing incentive

- Welfare-based social relations (caring, providing)

- Government as distribution mechanism

There were too few mentions of near equality (NREQ) to build a similar list.

Table 5

Distribution Rules Observed and Endorsed for Income

Observed $^{\mathrm{a}}$

Endorsed $^{\mathrm{a}}$

Proportionality

95.0

20.3

Near equality

10.1

82.3

Equality

1.7

11.8

Need

10.5

81.4

\footnotetext{
${ }^{\mathrm{a}}$ Numbers in these columns $=$ percentage of respondents who agree or strongly agree.
} 
Table 6

Observation of Mixed Rule Use for the Distribution of Income

Observed $^{\mathrm{a}} \quad$ Endorsed $^{\mathrm{a}}$

Proportionality only

81.7

Need only

Proportionality and near equality, equality, or need

10.0

Proportionality, need, and near equality or equality

One or both of near equality and equality

Near equality and need

Near equality, equality, and need;

0

\footnotetext{
${ }^{\mathrm{a}}$ Numbers in these columns $=$ percentage of respondents who agree or strongly agree.
}

within a reasonable range") is notable. This finding contrasts with Frohlich, Oppenheimer, and Eavey's (1987) finding that subjects tended to reject a range constraint, although such questions are very sensitive to the wording of questions.

When multiple rule use is considered, the most important combination is near equality and need, which received support from a majority of the sample (see Table 6). When other egalitarian combinations are included, that majority rises to 71.3 percent. This constitutes a definite endorsement of a less unequal society.

For the descriptions of society following the section on the distribution of income, there are significant associations between the descriptive categories observed and the distribution rules observed, but not between descriptive categories observed or endorsed and any of the rules endorsed. Although there is little difference in the descriptions of society, respondents are more likely to agree that there are cooperative relations if they think near equality is used to distribute income and more likely to endorse nurturant relations if they think need is used. In other words, people who perceive the use of different distribution rules go on to perceive society in different ways. All of these associations are significant at $\mathrm{p}<.0001$.

If one attempts to use the most frequently occurring subcategories for each rule to build up idea sets, a rather different picture emerges (see Table 7). This 
Table 7

The Most Frequently Used Categories by Those Observing the Three Distribution Rules for Income

\begin{tabular}{|c|c|c|c|}
\hline & Proportionality & Near Equality & Need \\
\hline Group & Hierarchical & Competitive & Competitive \\
\hline Relations & Interdependent & Exchange & Individualistic \\
\hline $\begin{array}{l}\text { Individuals } \\
\text { (second choice) }\end{array}$ & $\begin{array}{l}\text { Individuals } \\
\text { with basic needs } \\
\text { (competitive } \\
\text { and needing } \\
\text { motivation) }\end{array}$ & $\begin{array}{l}\text { Individuals } \\
\text { with basic needs } \\
\text { (competitive, } \\
\text { needing motivation, } \\
\text { or caring) }\end{array}$ & $\begin{array}{l}\text { Individuals } \\
\text { with basic } \\
\text { needs (needing } \\
\text { motivation) }\end{array}$ \\
\hline $\begin{array}{l}\text { Distribution } \\
\text { mechanism }\end{array}$ & Individual action & Individual action & Individual action \\
\hline
\end{tabular}

is essentially an individualistic image of society, centering on competitive and individualistic relations but seeing individuals as having basic needs. This convergence is probably due, at least in part, to the fact that respondents were free to agree with any combination of the distribution rules. This caveat notwithstanding, this does bear out the hypothesis that perceptions of distributions and perceptions of the group are significantly associated.

Different patterns of rule use and endorsement are found for other resources and other domains. Need is endorsed for healthcare by 91 percent of this sample, and only 12 percent use proportionality in any form. Moreover, 53.6 percent endorse equality and need. Job opportunities are thought to be distributed according to proportionality by 91.3 percent of the respondents, and 59 percent think proportionality alone is used. In the family, people were asked which of the distribution rules were used for two resources- "presents and material things" and "help, time, and effort"- both identified from the qualitative data. Although need is salient for both resources (65.6 percent and 76 percent, respectively) near equality is the most used rule for material goods ( 79.2 percent), whereas need is first and equality second for help, time, and effort (68.8 percent).

When the descriptive categories that agree with different resources are examined, the dominant image is still one of a hierarchical society with individualistic/competitive relations. Substantial numbers of respondents, however, also agree with more cooperative, community-oriented descriptions. It is as if 
there is a dominant view of society, but other attitudes held by substantial minorities of the population also exist. The need-based and egalitarian justice ideologies of the qualitative data seem more diffusely distributed in a larger population.

\section{General Discussion}

This study shows clear evidence for the association of relationshipdescribing attitudes and distribution rules. In the qualitative data, they occur in idea-sets, which look like the justice ideologies predicted by earlier work in that they provide information about the distribution, the recipient, and distribution mechanism. They also demonstrate a general orientation (competitive/individualistic, cooperative, and nurturant), which can be characterized in terms of Deutsch's $(1985,1987)$ motivational orientations or Fiske's (1991) relational orientations. In addition, they suggest that the three different orientations are qualitatively different: for example, descriptions of individuals are used much less with need than the other rules. However, examination of the categories of descriptions of individuals shows that where need has few such descriptions, proportionality is associated with differentiated individuals and equality with seeing people as similar. In this respect, they echo Lerner's (1991) developmental sequence of identity, unit, and nonunit relationships.

In the qualitative data, the actual idea-sets quite closely resemble the traditional left- and right-wing ideologies of equality/solidarity and market individualism. Fiske's perspective - that the relationship schemata give rise to expectations about the appropriateness of certain distribution rules as well as the standards by which any extant distribution is judged - would lead us to expect a dialectical relationship between what goes on in individual heads (patterned by the operation of relationship schemata) and what goes on in the cultural milieu (produced by individuals operating with the relationship schemata). The four-orientation model, however, received little support. Rather, these data follow the traditional three rules used in justice research over the last twentyfive years. The survey data, on the other hand, were less amenable to this process of identifying the most frequently used data, partly because respondents could choose more than one rule (see below) and partly because, whereas in the interview respondents only put forward those ideas that occurred to them at that time, in the survey each respondent was presented with the entire range of possible responses.

Both qualitative and survey data demonstrate that different rules are used for different resources within the socially constituted domains of society, work, local community, and so on. The variation is considerable and must surely cast doubt on the wisdom of looking for a rule applicable to a domain. Proportionalityeven in the reduced form of near equality - is supported for income, whereas need 
is endorsed for health care. This would seem to reflect experience of the British health service, which is free at the point of use and with which there is associated an explicit principle of need. Even within the family, different resources are not perceived to be allocated according to precisely the same rules.

The survey data also demonstrate that these British respondents have a strong streak of egalitarianism. A substantial majority endorses some form of reduced differentials, in spite of the fact that inequality increased markedly in the ten to fifteen years prior to the time of the survey. The wording of the question was: "The income people get does/should vary but within a reasonable range." The next step would be to see how great a range of incomes people would be prepared to support and whether their support would extend to an explicit cap on earnings, which has been rejected elsewhere (Frohlich and Oppenheimer 1992).

The survey data showed associations between observed distribution rules and observed characteristics of the society or group. Although the actual differences in patterns of attitudes were small, it must be remembered that respondents could choose any combination of the four rules they wished. This meant that there were combinations chosen that were contradictory, and led to a lack of discrimination between the attitudes associated with any one rule. This policy was chosen because there were so little data on British respondents at the time of the study that it seemed preferable to give the respondents more options, in the hope of remaining open to patterns that might have been missed in a forced-choice situation.

The finding that attitudes toward society varied with the principle of justice observed and not the ones endorsed, however, is reminiscent of the findings by Deutsch (1985) and Frohlich and Oppenheimer (1992) that distribution rules may be seen as fair even in the face of the subject's prior belief. It suggests that it is the distribution in which we participate that is the important determinant of our attitudes toward society and the social relationships we experience.

\section{ENDNOTE}

*Direct correspondence to: Department of Social Psychology, London School of Economics, Houghton St., London WC2A 2AE, UK, e-mail: r.e.stock@1se.ac.uk.

\section{REFERENCES}

Abric, Jean-Claude, and J. P. Kahan. 1972. "The Effects of Representations and Behaviour in Experimental Games." European Journal of Social Psychology 2:129-44. 
Cohen, Ronald L. 1987. “Distributive Justice: Theory and Research.” Social Justice Research 1:1940.

Desmarais, S., and Melvin J. Lerner. 1994. "Entitlements in Close Relationships." Pp. 43-64 in Entitlement and the Affectional Bond: Justice in Close Relationships, edited by Melvin J. Lerner and Gerold Mikula. New York: Plenum Press.

Deutsch, Morton. 1985. Distributive Justice. New Haven, CT: Yale University Press.

- 1987. "Experimental Studies of the Effects of Different Systems of Distributive Justice." Pp. 154-64 in Social Comparison, Social Justice, and Relative Deprivation, edited J. C. Masters and W. P. Smith. Hillsdale, NJ: Erlbaum.

Fiske, Alan P. 1991. The Structure of Social Life. New York: The Free Press.

Frohlich, Norman, and Joe A. Oppenheimer. 1992. Choosing Justice. Berkeley: University of California Press.

Frohlich, Norman, Joe A. Oppenheimer, and Cheryl L. Eavey. 1987. "Laboratory Results on Rawls's Distributive Justice." British Journal of Political Science 17:1-21.

Hochschild, Jennifer. 1981. What's Fair? American Beliefs about Distributive Justice. Cambridge, MA: Harvard University Press.

Kayser, Egon, and Thomas Schwinger. 1982. "A Theoretical Analysis of the Relationships among Individual Justice Concepts, Laymen's Social Psychology, and Distribution Decisions.” Journal for the Theory of Social Behaviour 12:47-51.

Kellerhals, Jean, Josette Coenen-Huther, and Marianne Modak. 1987. "Stratification Sociale, Types d'Interaction dans la Famille et Justice Distributive." Revue Francaise de Sociologie 28:21740.

- 1988. "Justice Norms and Groups Dynamics: The Case of the Family." International Sociology 3:119-27.

Kelley, J., and M. D. R. Evans. 1993. "The Legitimation of Inequality: Norms on Occupational Earnings in Nine Nations." American Journal of Sociology 99:75-125.

Lerner, Melvin J. 1991. "Integrating Societal and Psychological Rules of Entitlement." Pp. 13-32 in Social Justice in Human Relations, vol. 1, "Societal and Psychological Origins of Justice," edited by R. Vermunt and H. Steensma. New York: Plenum.

Matthews, Sarah H. 1987. "Perceptions of Fairness in the Division of Responsibility for Old Parents." Social Justice Research 1:425-37.

Skitka, Linda J., and Philip E. Tetlock. 1992. "Allocating Scarce Resources: A Contingency Model of Distributive Justice." Journal of Experimental and Social Psychology 28:491-522.

Tornblom, Kjell Y. 1992. "The Social Psychology of Distributive Justice.” Pp. 177-236 in Justice: Interdisciplinary Perspectives, edited by K. R. Scherer. Cambridge, UK: Cambridge University Press.

Tornblom, Kjell Y., and Uriel G. Foa. 1983. "Choice of a Distribution Principle: Cross-Cultural Evidence on the Effects of Resources." Acta Sociologica 26:161-73.

Tornblom, Kjell Y., and Dan R. Jonsson. 1987. "Distribution versus Retribution: The Perceived Justice of the Contribution and Equality Principles for Cooperative and Competitive Relationships." Acta Sociologica 30:25-52. 